\title{
FALLS IN NONAGENARIANS LIVING IN THEIR OWN HOMES: THE NONASANTFELIU STUDY
}

\author{
F. FORMIGA, A. FERRER¹, E. DUASO², C. OLMEDO'1, R. PUJOL
}

\begin{abstract}
Geriatric Unit, Internal Medicine Service, Hospital Universitari de Bellvitge, L’Hospitalet de Llobregat, Barcelona, Spain; 1. Primary Healthcare Center "El Plà" CAP-I, Sant Feliu de Llobregat, Barcelona, Spain; 2. Geriatric Unit, Internal Medicine Service, Hospital Mutua de Terrassa, Barcelona, Spain. Author Correspondence: Francesc Formiga, Geriatric Unit, Internal Medicine Service, Hospital Universitari de Bellvitge, L'Hospitalet de Llobregat, 08907 Barcelona, Spain, e-mail: fformiga $\Theta_{\mathrm{csub}}$.scs.es,
\end{abstract}

$$
\text { Tel: }+349326074 \text { 19; Fax: }+34932607420
$$

\begin{abstract}
Objectives: To evaluate the prevalence of falls and their circumstances in non-institutionalized people older than 89 years and living in an urban community. Design: Cross-sectional cohort study. Setting: Community-based study. Participants: 137 nonagenarians living at home. Measurements: We evaluated sociodemographic data, capacity to perform basic activities according to the Barthel Index (BI) and instrumental activities on the Lawton-Brody Index (LI), cognition with the Spanish version of the Mini-Mental State Examination (MEC), near visual acuity by the Snellen test, and auditory acuity with the whisper test. Results: Ninety-nine women $(72 \%)$ and 38 men with an average age of 93.07 years $(0.7)$ were included. $48.1 \%$ of them had suffered a fall during the last year, and in $20 \%$ of cases this had happened on more than one occasion. In $5.7 \%$ of cases, falls led to fractures. Factors associated with falls were a lower LI and a greater number of prescribed drugs. In the multivariate analysis the only factor related to falls was the number of drugs taken ( $p>0.001$, odds ratio $0.785,95 \%$ confidence interval $0.676-0.912$ ). Conclusions: Measures to prevent falls among nonagenarians should be intensified due to their high frequency. In this age group the increase in the percentage of falls is mainly related to the higher number of drugs taken.
\end{abstract}

Key words: Nonagenarians, functional capacity, falls, drugs.

Falls and fall-related fractures are a geriatric syndrome of great importance in the oldest old due to their frequency and the associated morbidity and cost. Indeed, falls may be one of the most important indicators for identifying frailty (1-2). In the elderly population a hip fracture is one of the most notable complications of falls (3), and most such fractures are secondary to a fall (1).

Approximately one third of elderly people living in the community experience at least one fall each year, and the number of falls increases progressively with age (2). Most falls have a multifactor etiology (1-3), and those people who have had a previous fall are at risk of falling again (2). In addition, elderly people often experience "post-fall syndrome", which generates the fear of falling again (4). The progressive aging of the population may lead to an increase in the number of falls. Very old people, such as nonagenarians, are frail and any fall can produce important complications such as a loss of functional capacity (5).

The purpose of this study was to estimate the frequency and possible consequences of falls reported in a cohort of community-dwelling elderly people aged 89 years old or more.

\section{Methods}

The study was conducted within the framework of the NonaSantfeliu study, a population-based study of nonagenarian inhabitants of Sant Feliu de Llobregat (Barcelona, Spain). This is an urban town of 45,000 inhabitants, who are mixed with regards to age and socio-economic status. All 305 persons older than 89 years old were contacted and asked to participate in the Received July 26, 2006

Accepted for publication February 16, 2007 study between January and April 2004. Contact was first made by letter and then confirmed by telephone. One hundred and nineteen of the total cohort could not be evaluated: 30 of them had moved to live with different relatives, 70 could not be traced because of incomplete data, 10 had already died and 9 refused to participate. The remaining $186(61 \%)$ agreed to take part in the study. Not residing in the community (49) was the only exclusion criterion, and subjects were not excluded according to health or cognitive status. Participants did not differ significantly from non-participants in terms of age or gender. The institutional ethics committee approved the study, and all patients or the caregivers of cognitively-impaired subjects gave their written informed consent before enrolment. Every inhabitant older than 89 years was interviewed in their home, rest home or health centre by a geriatric-trained research team. The geriatric assessment covered socio-demographic data (gender, marital status, education, and main caregiver), chronic diseases (stroke, dementia, diabetes), total number of falls, and the use of prescribed benzodiazepines.

\section{Overall geriatric assessment}

Functional, cognitive and nutritional status and social risk were assessed by instruments currently used in geriatric practice. Functional status was measured using the Barthel Index (6) (BI) for basic activities of daily living (ADL), while the Lawton and Brody Index (7) (LI) was used for instrumental ADL. The total BI score ranges from $0-100$ points (from help needed to independent in all activities). The LI scale ranges from $0-8$ points ( 8 meaning that no help is needed). Cognitive function was measured by the Spanish version of the Mini- 


\section{FALLS IN NONAGENARIANS}

Mental State Examination (MEC) [8], which yields a score out of 35 (a score of 23 or below indicates cognitive impairment).

\section{Sensory status}

Near sighted vision was measured with the equivalent Snellen charts, and a score lower than $20 / 40$ at $40 \mathrm{~cm}$ with the best eye and wearing the usual glasses was considered pathologic. For hearing competence the whisper test was used; this was performed $60 \mathrm{~cm}$ behind the subject's ear so that the examiner's lips could not be read (9). Visual and hearing difficulties were dichotomized as present or absent.

\section{Comorbidity}

The Charlson Comorbidity Index was used to measure overall comorbidity (10). This scale ranges from 0 to a theoretical maximum of 33 , depending on the presence of certain diseases with assigned scores.

\section{Orthostatic hypotension}

Blood pressure was determined using a mercury sphingomanometer, properly calibrated at the beginning of the study. The presence of orthostatic hypotension was defined as a decrease equal to or greater than $20 \mathrm{mmHg}$ in systolic blood pressure, or equal to or greater than $10 \mathrm{mmHg}$ in diastolic blood pressure after 1 to 3 minutes in the upright position, and with respect to the last measurement taken while standing.

\section{Fall assessment}

The main dependent variable evaluated was the presence of falls. A fall was defined as any incident in which the patient ends up on the ground or at a lower level against his/her will (not due to an intentional movement). A fall was not recorded if the person stopped against a piece of furniture, wall or other such structure (11). Falls due to an overwhelming force, such as a motor vehicle accident or a seizure were excluded. Patients were asked about any previous falls in the last year, including how many. This information was checked with the caregiver in all subjects with impaired memory. Based on fall outcomes, subjects were classified as either fallers or non-fallers. We also enquired about any architectural barriers in households: absence of an elevator or presence of outdoor stairs (four or more steps) leading to their building, or a house in which their bedroom was on the first floor (indoor stairs).

\section{Data analysis}

Normally-distributed continuous variables are reported as mean \pm standard deviation (SD). Categorical variables are reported as proportions. In bivariate analyses, the Student's T test was used to compare normally-distributed continuous variables, while the chi-squared statistic or Fisher's exact test were employed for the comparison of categorical or dichotomous variables. Multiple logistic regression analysis was performed to analyze the degree of statistical significance found $(p<0.05)$ in the bivariate analysis, as well as the influence of age and gender. SPSS 11.0 statistical software (SPSS Inc,
Chicago, III) was used for all analyses.

\section{Results}

The sample comprised 99 women $(72 \%)$ and 38 men, with a mean age of 93.07 years $(0.7)$. The majority $(n=115 ; 84 \%)$ were widowed, while $15(11 \%)$ were married and $7(5 \%)$ unmarried. A relative was the main caregiver in the case of 110 habitants $(80.3 \%)$. One percent had a university degree, $6.5 \%$ had reached high school, $63.5 \%$ had primary studies and $29 \%$ had received no formal education. In $60(44 \%)$ of the cases some kind of architectural barrier was present in the household: 40 lived in houses with indoor stairs and 20 on apartment block floors reached by outdoor stairs (without an elevator).

Table 1 shows socio-demographic differences between the two groups (falls in the previous year or not).

Table 1

Socio-demographic differences between the groups according to the presence or not of a fall.

\begin{tabular}{llll}
\hline & Fallers $(\mathbf{n = 6 6})$ & Non-fallers $(\mathbf{n = 7 1})$ & $\mathbf{p}$ \\
\hline Age & $92.8(38)$ & $93.2(3.1)$ & 0.70 \\
Female & $52(78 \%)$ & $47(66 \%)$ & 0.14 \\
Widowed & $56(85 \%)$ & $59(83 \%)$ & 0.96 \\
Married & $7(10.5 \%)$ & $8(11 \%)$ & 0.88 \\
Unmarried & $\mathbf{3 ( 4 . 5 \% )}$ & $\mathbf{4}(5.5 \%)$ & 1 \\
No education & & & \\
14 $(21 \%)$ & $26(36.5 \%)$ & 0.22 & \\
Primary & $46(70 \%)$ & $41(58 \%)$ & 0.20 \\
High school & $6(9 \%)$ & $3(4 \%)$ & 0.30 \\
University degree & 0 & $1(1.5 \%)$ & 1 \\
\hline
\end{tabular}

Assessment of instrumental activities yielded a mean LI score of 2.5 (2.3). With respect to basic activities of daily living, the mean BI score was 65.3 (28). As regards cognition, the mean MEC score was 22 (11).

The mean number of drugs taken was 4.2 (2.4), and 76 of the nonagenarians assessed were treated with more than 3 drugs $(55 \%)$. Thirty-seven nonagenarians $(27 \%)$ took benzodiazepines chronically. Diabetes (DM) was recorded in $19(14 \%)$, stroke in $27(20 \%)$, orthostatic hypotension in 17 $(12.5 \%)$ and dementia in $25(18 \%)$. Uncorrected auditory impairment was found in 56 cases $(41 \%)$, while near visual acuity was impaired in 51 cases $(37 \%)$. Combined sensory impairment was present in $34(25 \%)$ nonagenarians.

Table 2 shows the differences between the two groups (those with falls versus those without) and reveals a significant association between lower LI scores and a greater number of drugs being taken in the group of fallers. 


\section{THE JOURNAL OF NUTRITION, HEALTH \& AGING(C)}

Table 2

Differences between the groups according to the presence or not of a fall

\begin{tabular}{llll}
\hline & Fallers $(\mathbf{n}=66)$ & Non-fallers (n=71) & P \\
\hline Barthel Index & $63(27)$ & $67(28)$ & 0.35 \\
Lawton-Brody Index & $2.1(2.1)$ & $2.9(2.4)$ & 0.047 \\
MEC* & $22.6(11.4)$ & $22.4(11.1)$ & 0.91 \\
Charlson Comorbidity Index & $1.43(1.5)$ & $1.2(1.8)$ & 0.46 \\
Impairment in visual acuity & $30(45.5 \%)$ & $21(29.5 \%)$ & 0.08 \\
Impairment in hearing acuity & $28(42.5 \%)$ & $28(39.5 \%)$ & 0.86 \\
Combined sensory impairment & $16(24 \%)$ & $18(25 \%)$ & 0.96 \\
Dementia & $14(21 \%)$ & $11(15.5 \%)$ & 0.14 \\
Total number of drugs & $4.8(2.6)$ & $3.3(2.2)$ & 0.001 \\
Taking more than three drugs & $41(62 \%)$ & $35(49 \%)$ & 0.18 \\
Benzodiazepine use & $19(29 \%)$ & $18(25 \%)$ & 0.79 \\
Diabetes & $11(16.5 \%)$ & $8(11 \%)$ & 0.5 \\
Stroke & $18(27 \%)$ & $9(11.5 \%)$ & 0.05 \\
Orthostatic hypotension & $11(17 \%)$ & $6(8.5 \%)$ & 0.23 \\
\hline
\end{tabular}

* MEC: Spanish version of the Mini-Mental State Examination

\section{Falls and associated circumstances}

Sixty six $(48.1 \%)$ of the nonagenarians had had at least one fall during the previous year, and $27(20 \%)$ had fallen on more than one occasion. A total of 122 falls were recorded, with a mean of $1.8(1.2)$ falls per subject. In seven cases $(5.7 \%)$ the fall led to a bone fracture: 5 cases of hip fracture, one proximal humerus and one a pubis rupture. Of the 7 patients with a fracture two of them were receiving chronic benzodiazepine therapy $(5.5 \%$ of the total of 37 patients being treated with benzodiazepines), but no significant differences were found between these two and the five patients $(5 \%)$ with a fracture who were not taking these drugs $(p=0.99)$. In the logistic regression analysis in which the scores shown to be associated in the bivariate analysis were included (LI and number of drugs), the only factor that was independently related to falls in the previous year was the number of drugs taken $(p>0.001$, odds ratio $0.785,95 \%$ confidence interval $0.676-0.912$ ).

\section{Discussion}

The NonaSantfeliu study confirms the presence of a higher percentage of women and widowers in nonagenarians, and highlights that in our area an important number of noninstitutionalized inhabitants have to deal with architectural barriers. When evaluating falls among these communitydwelling nonagenarians, it is noteworthy that a large percentage (practically half of them) had fallen in the previous year.

Few studies have evaluated falls among the oldest old. Some authors have reported that female gender is associated with a greater number of falls (2). In the NonaSantfeliu study, this tendency among nonagenarians was also observed, although statistical significance was not reached, possibly due to the sample size and the smaller relative percentage of men. These factors, added to the fact that distance vision was not quantified (only near visual acuity was assessed), could explain the lack of association between uncorrected visual deficits and experiencing a greater number of falls.

Between 3 and $10 \%$ of falls lead to a bone fracture, hip fracture being the most frequent and severe (2). In the present sample of elderly patients a similar percentage of serious falls $(5.7 \%)$ was found, although in the majority of cases these did not lead to important physical damage.

One of the factors most frequently associated with an increased risk of falling is drug intake, although studies tend to focus on certain drug groups (12), such as benzodiazepines, rather than total drug consumption $(13,14)$. It is important to note that almost a third of the nonagenarians included in the present study had been taking benzodiazepines chronically.

Some studies in elderly people have reported an association between benzodiazepine use and falls leading to hip fracture. However, when plasma levels of benzodiazepines were checked this association was only confirmed for lorazepam (15), and the risk seems to be more important during the first two weeks after the start of treatment, after which it appears to decline (16). In our study, we observed no association between benzodiazepines and the number of falls, or with hip fractures.

The association between lower LI scores and a higher number of falls seems logical. People with greater capacity for instrumental activities in daily living are also likely to maintain a better level of physical activity, this being a known protective factor against falls (17).

Although orthostatic hypotension is generally considered to be a risk associated with a greater number of falls, this association has not been confirmed by many studies, such as the present one in non-institutionalized nonagenarians. This lack of association may be because the conventional diagnosis of orthostatic hypotension is less precise. When the diagnosis of "changes in arterial blood pressure" is evaluated by means of an "upright tilt" test, an association between orthostatism and a greater number of falls has been observed (18).

Although previous research has reported that elderly people with cognitive decline or dementia are at greater risk of falls (19), our study failed to confirm this, either with respect to MEC scores or a prior diagnosis of dementia. Neither was there a relationship with a previous history of stroke, although we did observe a greater - but non-significant - percentage of falls. Patients with diabetes have been reported to fall more of ten (20), and although such a trend was found in our population of nonagenarians it did not reach statistical significance.

Despite the strengths of this study, several limitations should be considered when interpreting the findings. The main limitation is the sample size, particularly in terms of the number of males. Another limitation was the failure to assess a number of common and important potential risk factors for falls, such as abnormalities of gait, balance or muscle weakness, etc. Moreover, as it is a cross-sectional study a clear temporal relationship between cause and effect cannot be established; this is the case of benzodiazepine use, as we did not record whether nonagenarians had taken the drug prior to the moment 


\section{FALLS IN NONAGENARIANS}

of the fall and neither were checks carried out of serum benzodiazepine levels. There may also be a memory bias affecting data collection given that the study relies on selfreports: elderly subjects were themselves asked about falls during the last year and they may not wish to acknowledge falls in order not to lose their sense of autonomy. At all events we believe this factor would affect the most serious falls, and is unlikely given the high percentage of falls detected and the fact that information was checked with caregivers.

It is highly important to establish the cause of a fall, at all healthcare levels, in order to implement measures that reduce the intrinsic and extrinsic risk factors involved and thus avoid future falls. Combined intervention programs aimed at several risk factors have been shown to be effective in preventing fractures (21). Technical assistance and, in selected patients, the use of hip protectors (22) can be very useful. The likelihood that a fall will lead to a fracture is greater among osteoporotic patients, and thus improved treatment for osteoporosis would be useful in reducing the percentage of fractures after a fall (23).

In conclusion, we observed a high frequency of falls among non-institutionalized nonagenarians and this suggests that preventive measures in this age group should be stepped up.

\section{References}

1. Formiga F, Rivera A, Nolla JM, Pujol R. Characteristics of falls producing hip fracture in an elderly population. Gerontology 2004; 50: 118-119.

2. Salvà $A$, Bolíbar I, Pera $G$, Arias $C$. Incidence and consequences of falls among elderly people living in the community. Med Clin (Barc) 2004: 122: 172-176

3. Tinetti ME, Speechley $M_{1}$, Ginter $S F$. Risk factors for falls among elderly persons living in the community. N Engl J Med 1988; 319: 1701-1707.

4. Murphy SL, Dubin JA, Gill TM. The development of fear of falling among community-living older women: predisposing factors and subsequent fall events. J Gerontol Med Sci 2003; 58A: M943-M947.

5. Formiga F, Lopez-Soto A, Sacanella E, Coscujuela A, Suso S, Pujol R. Mortality and morbidity in nonagenarian patients following hip fracture surgery. Gerontology 2003; 49: $41-45$.
6. Mahoney FI, Barthel DW. Functional evaluation: the Barthel Index. A simple index of independence useful in scoring improvement in the rehabilitation of the chronically ill. Md State Med J 1965; 14: 61-65.

7. Lawton MP, Brody EM. Assessment of older people: self-maintaining and instrumental activities of daily living. Gerontologist. 1969; 9:179-186.

8. Lobo A, Saz P, Marcos G, et al. Revalidación y normalización del Mini-Examen Cognoscitivo (primera versión en castellano del Mini-mental Status Examination) en la población general geriátrica. Med Clin (Barc) 1999; 112: 767-774.

9). MacPhee GJ, Crowther JA, Mc Alpine CH. A simple screening test for hearing impairment in elderly patients. Age Ageing 1988; 17: 347-351.

10. Charlson ME, Pompei $\mathrm{P}$, Ales KL, MacKenzie CR. A new method of classifying prognostic comorbidity in longitudinal studies: development and validation J Chronic Dis 1987; 40: 373-383.

11). Buchner DM, Hornbrook MC, Kutner NG, et al. Development of the Common Data Base for the FICSIT Trials. J Am Geriatr Soc 1993; 41: 297-308.

12. Weiner DK, Hanlon JT, Syudesnki SA. Effects of central nervous system polypharmacy on falls liability in community-dwelling elderly. Gerontology 1998 44: $217-221$.

13. Souchet E, Lapeyre-Mestre M, Montastruc JL Drug related falls: a study in the French Pharmacovigilance database. Pharmacoepidemiol Drug Saf. 2005; 14:11-16.

14. Kelly KD, Pickett W, Yiannakoulias N, Rowe BH, Schopflocher DP, Svenson L, Voaklander DC. Medication use and falls in community-dwelling older persons. Age Ageing 2003; 32: 503-509.

15. Pierfitte $\mathrm{C}$, Macouillard $\mathrm{G}$, Thicoïpe $\mathrm{M}$, et al. Benzodiazepines and hip fractures in elderly people: case-control study. BMJ 2001; 322: 704-708.

16. Wagner AK, Zhang $F$, Soumerai SB, et al. Benzodiazepine use and hip fractures in the elderly. Arch Intern Med 2004; 164: 1567-1572.

17. Gregg EW, Pereira MA, Caspersen CJ. Physical activity, falls, and fractures among older adults: a review of the epidemiologic evidence. J Am Geriatr Soc 2000; 48 883-893.

18. Heitterachi E, Lord SR, Meyerkort P, McCloskey I, Fitzpatrick R. Blood pressure changes on upright tilting predict falls in older people. Age Ageing 2002; 31: 181186.

19. Shaw FE. Falls in cognitive impairment and dementia. Clin Geriatr Med 2002; 18 159-73.

20. Wallace C, Reiber GE, LeMaster J, et al. Incidence of falls, risk factors for falls, and fall-related fractures in individuals with diabetes and a prior foot ulcer. Diabetes Care 2002; 25: 1983-1986

21. Tinetti ME, Baker DI, McAvay G, Claus EB, Garrett P, Gottschalk M. A multifactorial intervention to reduce the risk of falling among elderly people living in the community. N Engl J Med 1994; 331: 821-8244.

22. Kannues P, Parkkari J Niemi S, Pasanen M, Palvanen M, Järvinen M, Vuori I. Prevention of hip fracture in elderly people with use of hip protector. N Engl J Med 2000; 343: 1506-1513.

23. Formiga F, Rivera A, Nolla JM, Coscujuela A, Vivanco V, Sole A, Pujol R. Failure to treat osteoporosis and the risk of subsequent fractures in elderly patients with previous hip fracture: a five years follow-up study. Aging Clin Exp Res 2005; 17: 9699 